\title{
Right Testicular Seminoma in Bilateral Cryptorchidism: A Case Report
}

\author{
Ali Alamer ${ }^{\mathrm{a}, \mathrm{b}}$, Asim Aldhilan ${ }^{\mathrm{a}}$
}

\begin{abstract}
The incidence of testicular cancer in cryptorchidism is 30 to 50 times higher than general population. We report a case of a 48 -yearold male presents to our institute with abdominal pain. Huge mass was palpable within the right lower quadrant of the abdomen during local examination. CT scan examination of the abdomen and pelvis was performed and shows bilateral cryptorchidism with large right testicular mass. The left testicle is atrophied and located within the left aspect of the pelvis. Para-aortic lymph nodes metastasis is also identified.
\end{abstract}

Keywords: Cryptorchidism; Seminoma; Testicular mass

\section{Introduction}

The incidence of testicular cancer in cryptorchidism is 30 to 50 times higher than general population. We report a case of 48-year-old male with bilateral abdominal cryptorchidism and large retro peritoneum right testicular germ cell tumor associated with lymph nodes metastasis.

\section{Case Report}

A 48-year-old male patient presented to our institute with abdominal pain. No recent history of trauma. Clinical examination was performed and revelled a large and solid right lower abdominal quadrant mass. The scrotal sac was empty dur-

Manuscript accepted for publication March 13, 2013

${ }^{a}$ Medical Imaging department, King Abdulaziz Medical City, Riyadh, Saudia Arabia

${ }^{\mathrm{b}}$ Corresponding author: Ali Alamer, King Abdul Aziz Medical City, Riyadh, Saudia Arabia. Email: ali3meer@hotmail.com

doi: http://dx.doi.org/10.4021/wjnu75w ing examination. Vital signs, Complete blood count analysis, and blood chemistry were normal.

Computed tomography (CT) scan examination of the abdomen and pelvis was performed after administration of intravenous (IV) contrast material in portal venous phase which shows an empty scrotal sac with absence of the testicles and spermatic cord bilaterally (Fig. 1). A large homogenous mass is identified within the retro peritoneum of the right lower abdominal quadrant associated with small central area of low attenuation could represent an areas of cystic necrosis. No associated calcification is seen (Fig. 2). Small oval and homogenous structure is seen within the left aspect of the hemi pelvis just adjacent to the inguinal ring which represents a small atrophied left testicle with no masses (Fig. $3)$. There are multiple homogenous and matted lymph nodes metastasis located within the right Para-aortic region in close relation to the right renal hilum (Fig. 4).

The diagnosis was made to be right testicular germ cell tumor likely seminoma in bilateral cryptorchidism associated with Para-aortic lymph nodes metastasis for urology consultation. The differential diagnosis would include lymphoma and metastasis.

\section{Discussion}

Cryptorchidism is the most common genital problem encountered in pediatrics. Cryptorchidism literally means hidden or obscure testis and generally refers to an undescended or maldescended testis. The most common sites of cryptorchidism are high scrotal (50\%), canalicular $(20 \%)$ and abdominal (10\%), bilateral (10\%) [1]. The incidence of testicular cancer in cryptorchidism is 30 to 50 times higher than general population. The higher the testis is located (abdominal vs inguinal canal), the greater the risk of malignancy [1]. The risk is greatest in cases of bilateral cryptorchidism like our case [1]. Seminoma is the most common tumor in cryptorchidism. In general approximately $95 \%$ of malignant testicular tumors are germ cell tumors, of which seminoma is the most common histological subtype. Compared to the non-seminomatous germ cell tumors, seminoma occurs in an older patient population, with a mean age of approximately 


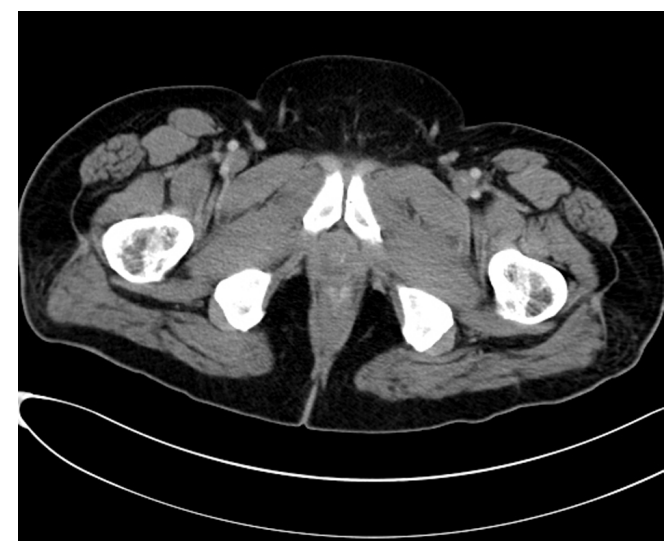

Figure 1. Computed tomography (CT) scan examination shows an empty scrotal sac with absence of the testicles and spermatic cord bilaterally.

40 years [2]. These tumors carry a favorable prognosis due to their sensitivity to radiation and chemotherapy [2].

Approximately $75 \%$ of patients with seminoma present with disease limited to the testis, $20 \%$ have retroperitoneal adenopathy, and 5\% have extra-nodal metastasis [3]. Check the retroperitoneal lymph node (especially at the level of renal hilum) in all cases of cryptorchidism complicated by malignancy like our case which presents by Para-aortic lymph nodes metastasis in close relation to the renal hilum. Absence of the spermatic cord is an important clue for cryptorchidism. Other differential diagnoses of retroperitoneal masses in adults include enlarged lymph nodes from lymphoma or infectious processes, neurogenic tumours, sarcoma and fibrosis.

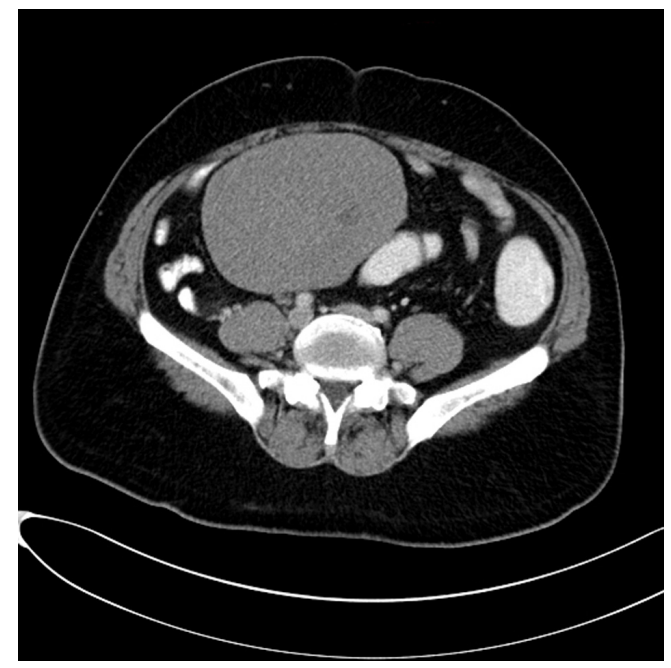

Figure 2. A large homogenous mass is identified within the retro peritoneum of the right lower abdominal quadrant associated with small central area of low attenuation could represent an areas of cystic necrosis. No associated calcification is seen.

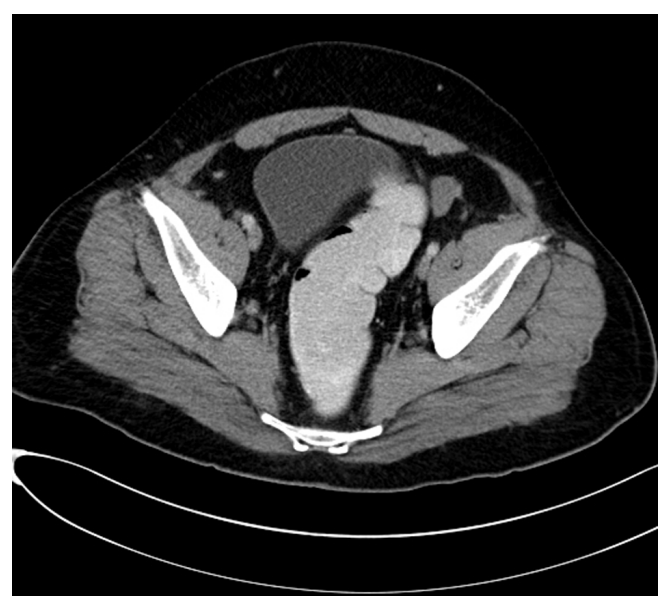

Figure 3. Small oval and homogenous structure is seen within the left aspect of the hemi pelvis just adjacent to the inguinal ring which represents a small atrophied left testicle with no masses.

Treatment of seminoma involves surgical removal of the testicular primary. Radiotherapy to regional nodes if there is local disease (stage I) or limited nodal Para-aortic metastases (non-bulky stage II). Chemotherapy if there is bulky Paraaortic lymph node involvement of more detent disease. Prognosis is good for all stages with greater than $90 \%$ cure rate.

To conclude, the most common sites of cryptorchidism is high scrotal, and the higher the testis is located, the greater the risk of malignancy. The risk is greatest in cases of bilateral cryptorchidism. Seminoma is the most common tumor in cryptorchidism. Check the retroperitoneal lymph node (especially at the level of renal hilum) in all cases of cryptorchidism complicated by malignancy.

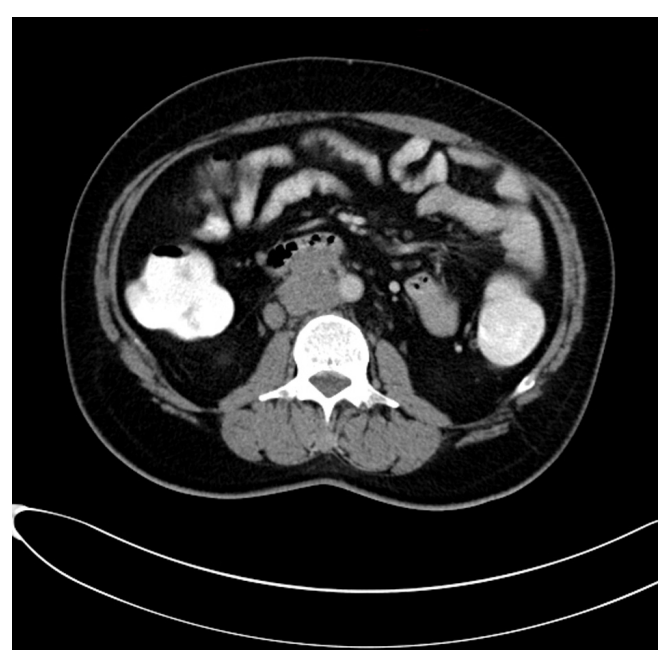

Figure 4. There are multiple homogenous and matted lymph nodes metastasis located within the right Para-aortic region in close relation to the right renal hilum. 


\section{References}

1. L.V. Chandrasekharan, T.F. Abdl Ghaffar, M. Venkatramana, S. Rath (2005) A Case Of Teratocarcinoma In Abdominal Testis. The Internet Journal of Radiology $10.5580 / 2347 .$.

2. Kim W, Rosen MA, Langer JE, Banner MP, Siegel- man ES, Ramchandani P. US MR imaging correlation in pathologic conditions of the scrotum. Radiographics. 2007;27(5):1239-1253.

3. Woodward PJ, Sohaey R, O’Donoghue MJ, Green DE. From the archives of the AFIP: tumors and tumorlike lesions of the testis: radiologic-pathologic correlation. Radiographics. 2002;22(1):189-216. 